\title{
MUICSER: A MULTI-DISCIPLINARY USER-CENTERED SOFTWARE ENGINEERING PROCESS TO INCREASE THE OVERAL USER EXPERIENCE
}

\author{
Mieke Haesen, Kris Luyten, Karin Coninx, Jan Van den Bergh and Chris Raymaekers \\ Hasselt University - $t U L$ - IBBT, Expertise Centre for Digital Media, Wetenschapspark 2, B-3590 Diepenbeek, Belgium \\ $\{$ mieke.haesen,kris.luyten,karin.coninx,jan.vandenbergh,chris.raymaekers\}@uhasselt.be
}

Keywords: User-Centered Design, Software Engineering, Model-Driven

\begin{abstract}
In this paper we present an incremental and user-centered process to create suitable and usable user interfaces. Validation is done throughout the process by prototyping, the prototypes evolve from low-fidelity to the final user interface. Applications developed with this process are more likely to correspond to users' expectations. Furthermore, the process takes into account the need for sustainable evolution often required by modern software configurations, by combining traditional software engineering with a user-centered approach. We think our approach is beneficial in its scope, since it considers evolving software beyond the deployment stage and supports a multi-disciplinary team.
\end{abstract}

\section{Introduction}

In this contribution we present an overall approach for the (re-)design of interactive applications that takes into account the needs of the end-user as well as the needs of the designer and developer. Our approach can be used to design new applications that require input from many different sources (e.g. domain experts) or even to redesign legacy applications to improve the user experience they offer. This approach builds on existing models and notations in both the software engineering and human-computer interaction (HCI) community, providing extensions where necessary.

Based on our experiences and observations when working with multi-disciplinary teams, we are gradually introducing an agile model-based approach in applied research and software development projects. We will be using different models throughout the process, where each model describes a specific aspect of an interactive system and represents the viewpoint of one or more specific roles in the multi-disciplinary team.

User-Centered Design (UCD) approaches are meant to support the development process in such a way that software quality attributes like usability and overall compliance to the user needs are taken into account. Therefore, combining a model-driven approach and a user-centered development process seems a natural way to ensure benefits for the enduser as well as the development team. The artifacts created using the proposed user-centered development cycle contribute to well-considered and traceable design decisions. The need for communication with end-users or customers results in additional models or artifacts (e.g. low-fidelity and high-fidelity prototypes) on top of the commonly used models in a model-driven approach, which are very helpful to support the communication in a multi-disciplinary project team (e.g. task model, presentation model etc.).

In this paper, we present a user-centered software engineering proces and detail the models and artifacts that are at the center of the approach. A discussion of our current and future work, as well as related work, conclusions are presented.

\section{MuiCSer}

Our Multi-disciplinary user-Centered Software engineering process, MuiCSer, embodies UCD with a structured Agile Software Engineering (ASE)((Larman, 2003)) process. Figure 1 gives an overview of the proposed process. At first this may seem as a waterfall type of process model. However, 


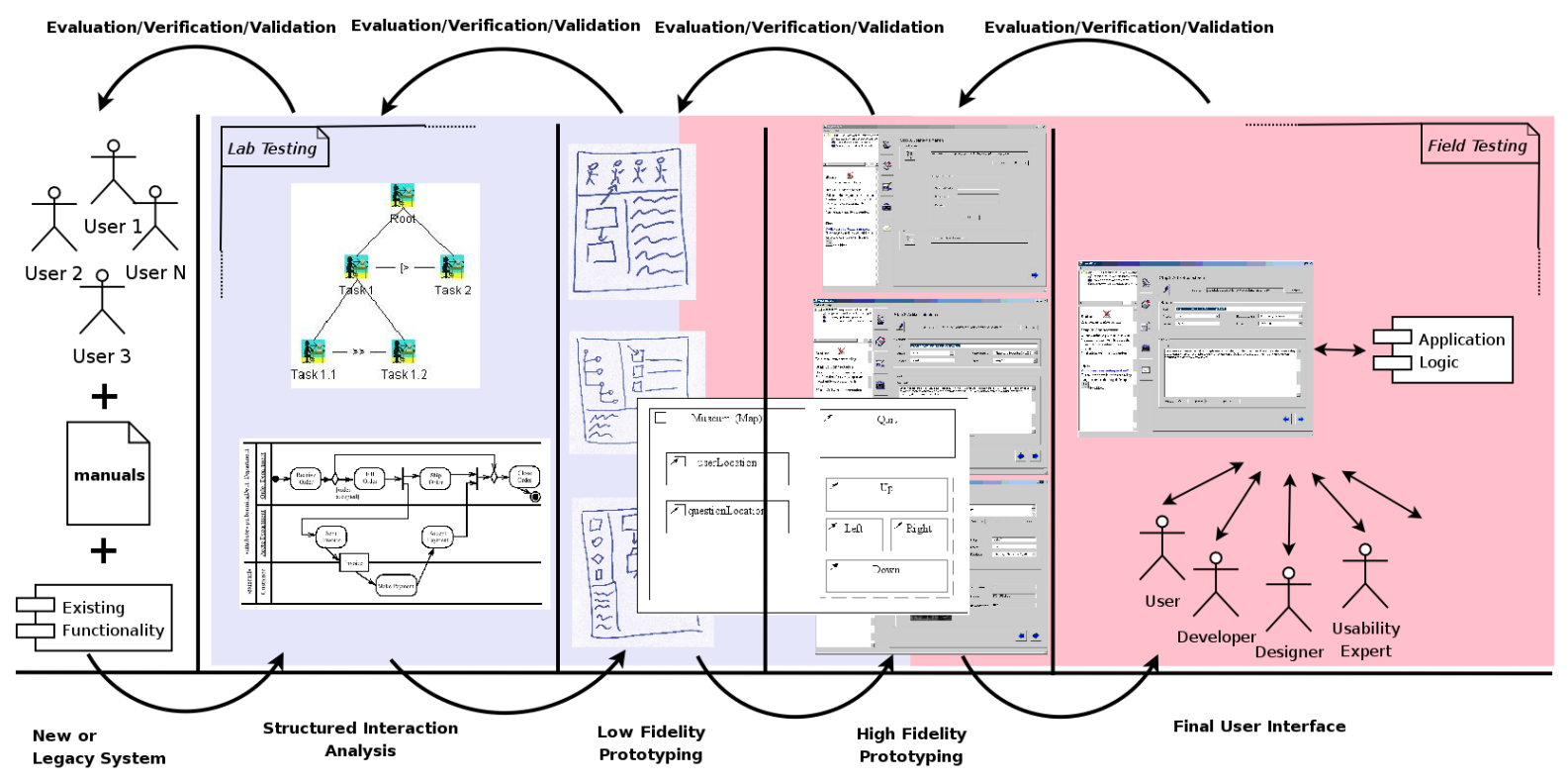

Figure 1: The MuiCSer Process.

the development team can choose which stages are tackled during one timeboxed iteration. At the end of each iteration, there will be artifacts which can be of various types (e.g. low-fidelity prototypes, UML diagrams, executable code,...) and will be stored in a central repository ready for use in the next iteration. The next paragraphs discuss the properties of the process we propose in more detail.

The process typically starts with an analysis phase in an initial iteration where the users tasks, goals and the related objects or resources that are important to perform these tasks are specified. If the user experience of a legacy system needs to be optimized, the functionality of such a system can be often found in existing manuals thus it can also contribute to the analysis.

During the structured interaction analysis, the results of the first stage are used to progress towards system interaction models and presentation models. These models are often expressed using the UML notation, thus being kept in pace with the traditional SE models.

Since both user needs and functional information are specified, they can serve as input for the low fidelity prototyping stage. User interface designers create mockups of the user interface, based on the information contained in the task and interaction models, while using design guidelines and their experience. In subsequent phases, we go from low-fidelity to high-fidelity prototypes, which on their turn evolve into the concrete user interfaces. So each stage grad-

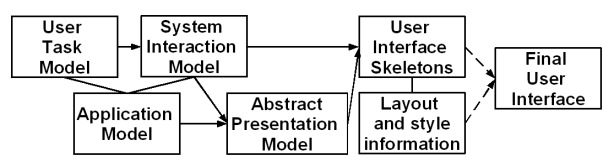

Figure 2: Artifacts created during the design process.

ually evolves towards the final user interface, but is still related to the artifacts created in a previous stage.

An overview of the artifacts involved can be seen in Figure 2. It shows the artifacts created or updated during the design and the relations between them.

By evaluating the result of each stage, the support for user needs and goals and the presence of required functionality is verified. If possible, an evaluation with target users can be very useful to get feedback from the end user directly. Because most of the artifacts do not present a fully functional system, part of the testing takes place in a usability lab. To evaluate some advanced prototypes, field tests can examine the user interface in more realistic situations. If the results of a phase are too complex to involve an end user during evaluation, it is still necessary to evaluate, verify or validate the models or prototypes.

MuiCSer as depicted in Figure 1 shows only one iteration (or increment) in the development of the complete system. The back arrows at the upper side of the figure emphasize that one can iterate over the previous phase, depending on the results of the evaluation of the current artifact. 


\section{HCI Engineering Models}

The proposed notations are built on existing model notations used in software engineering and interface design and specification. The following sections describe different models being created, changed and transformed during the execution of the process in order to support a smooth transfer towards the final user interface. These models and their interrelations are shown in the left part of Figure 2.

\subsection{The User Task Model}

User task models specify requirements for an application from a users's point of view, in a hierarchical way. We use the ConcurTaskTrees (CTT) notation (Mori et al., 2002) in the second stage of MuiCSer. It has proven to be easy to understand and usable, even for complex systems. In our previous work, we indicated that working prototypes with inter- and intradialog navigation can be generated by combining this notation with high-level user interface descriptions (Luyten et al., 2003).

Van den Bergh and Coninx (Van den Bergh and Coninx, 2006) showed a correspondence between the CTT notations and UML 2.0 activity diagrams. This mapping results in the system interaction model as described in section 3.3.

\subsection{The Application Model}

The application model is traditionally used to describe the objects and commands provided by the application logic. The model is represented using the UML class diagrams and can be considered most closely related to the data or object model. The application model is useful to describe the interface between the user interface and the application logic.

Furthermore, the application model is an important model for legacy systems, since the application logic is already available when the design of a (new) user interface starts.

\subsection{The System Interaction Model}

The system interaction model offers a system-centric description of action flows that can be performed by the system, by a user through the system, or that have an influence on the system. The model is expressed using UML 2.0 activity diagrams, extended with new stereotypes corresponding to the task types in the CTT notation.

\subsection{Abstract Presentation Model}

The abstract presentation model defines the structure of the user interface in a platform independent manner describing the logical and temporal structure of the user interface through a hierarchy of abstract interaction components. The model is expressed using the UML class diagram, in which each component is represented by a stereotyped class ${ }^{1}$.

A complete formal mapping to the Canonical Abstract Prototypes (CAP) (L. Constantine, 2003) is not possible due to the informal semantics, rudimentary rules were however identified. The notation will be used as a front-end for a designer to add additional information, such as screen organization.

In MuiCSer, we employ high-level user interface descriptions to define the high-fidelity prototype. We prefer UIML (Luyten and Coninx, ) and XForms (Van den Bergh et al., 2007) to render the prototypes, but other user interface description languages are also possible. These languages should be XML-based and independent of the application logic, to smooth the transformation between prototypes and the final user interface.

\section{Ongoing and Future Work}

The process presented in this paper has been tested on software projects of limited complexity and, by consequence, with a development team of limited size. Although our tests did not include any larger software projects, the process should be flexible enough to support the increased complexity and team size. One of our main contributions is that different domain experts can use their own notations to create models which can relate to models of other domain experts, in order to obtain a complete and usable interactive system with respect to the requirements. We are testing this approach in different application domains (applications for logistics, community websites etc.), requiring different experts to collaborate.

We plan to gradually improve the relation between the different types of artifacts, which is a key factor for success. The combination of HCI models and UML models guarantees a smooth integration of the user interface and application logic. Putting forward the combination of models explicitly also prevents mismatches between the functionality provided by the application logic and the functionality accessible through the user interface.

\footnotetext{
${ }^{1}$ Stereotypes (Object Management Group, 2004) are standardized means to extend the UML language.
} 


\section{Related Work}

This paper is not the first to look into the relation between user-centered design and both HCI and software engineering models. Hudson (Hudson, 2004) describes a way to use UML in combination with user-centered design, but does not address how user interfaces can explicitly be modeled in UML.

A study on the state of UCD by Mao et al. (Mao et al., 2005) showed among other things that although many parts of UCD are practiced in actual development in industry, end-to-end deployments are seldom due to limitations of current practice.

Wisdom (Nunes, 2001) defines a set of models using the standard UML-diagrams and stereotypes to create more appropriate notations for a variety of models that can be used to design interactive systems in small teams. Wisdom also provides a user-centered methodology to effectively use the notation.

Campos et al. (Campos and Nunes, 2005) proposed a workstyle model, Galactic Dimensions, that focuses on workstyle transitions rather than a design process. The workstyle model allows to evaluate tools according to different dimensions that characterize different workstyles.

\section{Conclusions}

In this paper we introduced a novel process, practicing User-Centered Software Engineering in such ways that methodologies used by developers as well as the creativity of designers are included and a positive user experience is more likely to be obtained. Although both HCI and Software Engineering models are integrated into one process, it is up to the multidisciplinary project team which models will be used. To provide even more flexibility, we also included explicit support for legacy systems being updated or extended.

Whenever possible, an evaluation with end users is recommended in each step. However since end users are not always available or should not understand all models, validation or verification by the project team is suggested. This approach also encourages the development of software in several iterations and increments.

\section{ACKNOWLEDGMENTS}

Part of the research at EDM is funded by the ERDF (European Regional Development Fund) and the Flemish Government. The VIP-lab project
(4-BMG-II-2=37), is financed by the "Interreg Benelux-Middengebied" authorities and co-financed by Province of Limburg (B), Province of Limburg (NL), Ministry of Economic Affairs (NL) and Ministry of Flemish Government/Economic Affairs (B).

The MuiCSer process is also based on our experiences in IWT projects Participate (with AlcatelLucent) and AMAS++ (IWT 060051).

\section{REFERENCES}

Campos, P. F. and Nunes, N. J. (2005). Galactic Dimensions: a Unifying Workstyle Model for User-Centered Design. In INTERACT'05, volume 3585 of LNCS, pages $158-169$. Springer.

Hudson, W. (2004). Object Modeling and User Interface Design, chapter Toward Unified Models in UserCentered and Object-Oriented Design, pages 313 362. Addison Wesley.

L. Constantine, L. (2003). Canonical abstract prototypes for abstract visual and interaction design. In Proc. DSV-IS 2003, volume 2844 of $L N C S$, pages 1-15. Springer.

Larman, C. (2003). Agile and Iterative Development: A Manager's Guide. Addison-Wesley.

Luyten, K., Clerckx, T., Coninx, K., and Vanderdonckt, J. (2003). Derivation of a Dialog Model from a Task Model by Activity Chain Extraction. In Proc. DSV-IS 2003, volume 2844 of $L N C S$, pages 203-217.

Luyten, K. and Coninx, K. Uiml.net: an open uiml renderer for the .net framework. pages 257-268.

Mao, J.-Y., Vredenburg, K., Smith, P. W., and Carey, T. (2005). The state of user-centered design practice. Communications of the ACM, 48(3):105-109.

Mori, G., Paternò, F., and Santoro, C. (2002). CTTE: support for developing and analyzing task models for interactive system design. IEEE Transactions on Software Engineering, 28(8):797-813.

Nunes, N. J. (2001). Object Modeling for User-Centered Development and User Interface Design: The Wisdom Approach. $\mathrm{PhD}$ thesis, Univ. da Madeira.

Object Management Group (2004). UML 2.0 Superstructure Specification.

Van den Bergh, J. and Coninx, K. (2006). Cup 2.0: Highlevel modeling of context-sensitive interactive applications. In Proc. MoDELS 2006, volume 4199 of LNCS, pages 140-154.

Van den Bergh, J., Huypens, S., and Coninx, K. (2007). Towards Model-Driven Development of Staged Participatory Multimedia Events. In Proc. DSV-IS, volume 4323 of LNCS, pages 81-94. 